\title{
Correlation of acidosis-adjusted potassium level and cardiovascular outcomes in diabetic ketoacidosis: a systematic review
}

This article was published in the following Dove Press journal: Diabetes, Metabolic Syndrome and Obesity: Targets and Therapy

\author{
Atif Usman' \\ Mohd Makmor Bakry ${ }^{2}$ \\ Norlaila Mustafa ${ }^{3}$ \\ Inayat Ur Rehman ${ }^{1,4}$ \\ Allah Bukhsh ${ }^{1,5}$ \\ Shaun Wen Huey Lee' \\ Tahir Mehmood Khan 1,5,6 \\ 'School of Pharmacy, Monash University, \\ Bandar Sunway, Selangor, Malaysia; \\ ${ }^{2}$ Faculty of Pharmacy, Universiti \\ Kebangsaan Malaysia, Kuala Lumpur. \\ Malaysia; ${ }^{3}$ Department of Endocrinology, \\ Universiti Kebangsaan Malaysia Medical \\ Centre, Kuala Lumpur, Malaysia; \\ ${ }^{4}$ Department of Pharmacy, Abdul Wali \\ Khan University, Mardan, Pakistan; \\ ${ }^{5}$ Institute of Pharmaceutical Sciences, \\ University of Veterinary and Animal \\ Sciences, Lahore, Pakistan; ${ }^{6}$ Asian Centre \\ for Evidence Synthesis in Population, \\ Implementation and Clinical Outcomes, \\ Health and Well-being Cluster, Global \\ Asia in the 21 st Century Platform, \\ Monash University Malaysia, Selangor, \\ Malaysia
}

Background: During the progress and resolution of a diabetic ketoacidosis (DKA) episode, potassium levels are significantly affected by the extent of acidosis. However, none of the current guidelines take into account acidosis during resuscitation of potassium level in DKA management, which may increase the risk of cardiovascular adverse events.

Objective: To assess literature regarding the adjustment of potassium level using $\mathrm{pH}$ to calculate $\mathrm{pH}$-adjusted corrected potassium level, and to observe the relationship of cardiovascular outcomes with reported potassium level and $\mathrm{pH}$-adjusted corrected potassium in DKA. Methodology: Seven databases were searched from inception to January 2018 for studies which had reported people with diabetes developing diabetic ketoacidosis, in relation to prevalence or incidence, fluid resuscitation or potassium supplementation treatment, treatment or cardiovascular outcomes, and experimentation with DKA management or insulin. Quality of studies was evaluated using Cochrane Risk of Bias and Newcastle Ottawa Scale. Results: Forty-seven studies were included in qualitative synthesis out of a total of 10,292 retrieved studies. Forty-one studies discussed the potassium level and blood $\mathrm{pH}$ at the time of admission, ten studies discussed cardiovascular outcomes, and only four studies concurrently discussed potassium level, $\mathrm{pH}$, and cardiovascular outcomes. Only two studies were graded as good on the Newcastle Ottawa Scale. The reported potassium level was well within normal range $(5.8 \mathrm{mmol} / \mathrm{L})$, whereas $\mathrm{pH}$ rendered patients to be moderately acidotic $(7.13)$. Surprisingly, none of the included studies mentioned $\mathrm{pH}$-adjusted corrected potassium level and, hence, this was calculated later. Although mean corrected potassium was within the normal range $(3.56 \mathrm{mmol} / \mathrm{L}), 13$ studies had corrected potassium below $3.5 \mathrm{mmol} / \mathrm{L}$ and five had it below $3.0 \mathrm{mmol} / \mathrm{L}$. Nevertheless, with the exception of one study, none discussed cardiovascular outcomes in the context of potassium or $\mathrm{pH}$-adjusted potassium level.

Conclusion: The evidence surrounding cardiovascular outcomes during DKA episodes in light of a $\mathrm{pH}$-adjusted corrected potassium level is scarce. A prospective observational, or preferably, an experimental study in this regard will ensure we can modify and enhance safety of existing DKA treatment protocols.

Keywords: diabetic ketoacidosis, potassium, hypokalemia, blood gasses, acidosis, pH, treatment outcomes, cardiovascular, insulin

\section{Background Diabetic ketoacidosis}

Diabetic ketoacidosis is a prevalent acute hyperglycemic complication of diabetes mellitus. ${ }^{1,2}$ It is a severe and life threatening complication, and requires immediate therapeutic interventions which may otherwise lead to a fatal outcome. ${ }^{3}$ The most
Correspondence: Atif Usman School of Pharmacy, Monash University Malaysia, Jalan Lagoon Selatan 47500 Email Atif.

Tahir Mehmood Khan

School of Pharmacy, Monash University Malaysia, Jalan Lagoon Selatan 47500,

Bandar Sunway, Selangor, Malaysia

Email tahir.mehmood@monash.edu; tahir.khan@uvas.edu.pk 
common causes of any DKA episode are poor compliance, infection, and physical stress, ie, cardiovascular (CV) disorder. ${ }^{2,3}$ Initial insulin deficiency causes hyperglycemia, ketonemia, and acidosis, all of which promote diuresis. This causes notable hypovolemia and loss of electrolytes. In response to the metabolic and hypovolemic stress, the concentration of counter regulatory hormones $(\mathrm{CRH})$, ie, catecholamines, increases significantly. Increase in CRH further exacerbates circulatory distress. ${ }^{4}$ Metabolic, hormonal, and circulatory impairment in DKA requires swift action. In this regard, recommended treatment of DKA encompass of correction of blood volume, achieving euglycemia, and restoration of normal $\mathrm{pH}$ utilizing intravenous fluid resuscitation, insulin, and bicarbonate buffer, respectively. ${ }^{4-7}$

\section{Potassium in diabetic ketoacidosis}

Hypokalemia is a frequently observed complication in DKA. The role of potassium during an episode of DKA is very crucial where the abovementioned factors have an arguable influence on its regulation. ${ }^{8-10}$ Initially, DKA patients experience hyperkalemia; insulin deficiency renders cellular inability to allow potassium re-entry into the cell, and catecholamines induced cellular insensitivity hinders cellular uptake of potassium. ${ }^{11}$ Upon further progress, acidosis causes desensitization of cognate receptors of insulin and catecholamines, resulting in exacerbating hyperkalemia. ${ }^{12}$ Nevertheless, hyperkalemia swiftly changes to hypokalemia. Primary renal clearance of excessive glucose, and secondary renal clearance of ketone bodies cause polyurea and hypovolemia. In order to maintain homeostasis, the kidneys retain sodium and bicarbonate at the expense of potassium excretion. ${ }^{10,13}$ Diuresis induced by hyperglycemia and ketosis alone depletes as much as $3-15 \mathrm{mmol} / \mathrm{kg}$ of potassium. ${ }^{14,15}$ Additionally, hyperkalemia-induced vomiting in DKA causes a loss of potassium too. ${ }^{16}$

\section{Potassium in cardiovascular outcomes}

Cardiovascular disorders are also among precipitating factors of DKA whereby they cause physical stress in DM patients. ${ }^{17}$ Among CV disorders, myocardial infarction (MI) is the most observed cardiac incident which triggers an episode of DKA. ${ }^{1-3,18}$ Moreover, the concentration of CRH increases significantly during acidosis, which further increases the risk of adverse CV outcome in DKA patients. Although an episode of MI may not be directly associated with potassium level, potassium remains an important repolarization electrolyte in the cardiac cycle. At any point of an episode of acute MI, the level of potassium is often considered crucial as the patient may also experience life threatening arrhythmia secondary to catecholamine driven influx of potassium ion. ${ }^{19,20}$ Physiologic effects of hypokalemia involving pulse induction are altered primarily due to changes in refractory periods of myocardia, and result in the form of premature ventricular contractions, fibrillation, and tachycardia. These effects are also visible when considering an electrocardiograph (ECG) in DKA patients; a relative flat ST with a prominent $U$ wave fusing with a $\mathrm{T}$ wave is observed among patients with DKA experiencing hypokalemia. ${ }^{21,22}$ This leads to the notion that ECG and CVS stability shall also be considered as prime objectives when treating a DKA patient.

\section{Rationale and objectives}

The consequences of hypokalemia accompanied by acidosis are relatively deleterious when physiologic cardiac conduction is hindered. ${ }^{19,23}$ Moreover, deterioration of a patient's condition may be observed when $\mathrm{pH}$ impairs the regular function of insulin and catecholamines. On the other hand, besides that acidosis is a major factor for hypokalemia in DKA, it also has CVS suppressive effects. ${ }^{11}$ Hydrogen ions reduce cardiac output and peripheral vasodilation, hinder inotropic effect, and cause bradycardia. Additionally, the association of outcomes of DKA patients with elevated cardiac troponin I, although not extensive, somehow depicts that CVS does undergo enormous stress during a DKA episode. ${ }^{24}$ All these effects of DKA on CVS encourage us to signify the effect of potassium on CV outcomes in light of acidosis in DKA patients. Hence, this systematic review aims to explore the difference between reported potassium level and $\mathrm{pH}$-adjusted corrected potassium level among the DKA patients. Second, it also focuses on reports of CV complications among DKA patients and its correlation with admission and $\mathrm{pH}$-adjusted corrected potassium level.

\section{Methodology}

A systematic review of the published literature was performed following the Preferred Reporting Items for Systematic Reviews and Meta-Analyses (PRISMA) guidelines. ${ }^{25}$ Seven databases - EBSCOhost, PubMed, Scopus, Ovid databases (Cochrane, Embase, and MEDLINE), and clinical trial registries - were searched from the date of inception until January 30, 2018. Main MeSH terms for DKA, adapted form Andrade-Castellanos 
$\mathrm{CA}$ et $\mathrm{al}^{26}{ }^{26}$ were as follows: "Diabetic ketoacidosis", "Diabetic Coma", and (Hyperglycaemic OR Hyperglycemic OR Diabet* AND (Keto* OR acidos* OR coma) OR Emergen*) OR DKA. Similarly, MeSH terms related to treatment, treatment modalities, the outcome of treatment, and potassium were adapted from Tran et al. ${ }^{27}$ Finally, terms for insulin and cardiovascular were added to conclude the search. All the terms were connected with relevant key words of varying management aspects of DKA (Supplement). Boolean operators were used to combine text terms, keywords, and MeSH terms. This was further supplemented by a backward and forward manual search of relevant references. Once finalized, search terms were evaluated by TMK, NM, IR, and AB.

\section{Registration of study protocol}

The protocol was registered with PROSPERO (Registration Number: CRD42018098772).

\section{Study selection}

An article was included if it met the following criteria: 1) the study reported the prevalence of DKA in adult diabetic patients and assessed admission potassium level and $\mathrm{pH}$; 2) the study qualitatively observed cardiovascular outcomes in DKA patients; 3) the design of the study was a cross-sectional or cohort, or randomized controlled trial. Studies were excluded if 1) it was a case-control, case report, conference proceeding, systematic review, letter to editor, an opinion, or research brief; 2) published in languages other than English; 3) reported DKA secondary to pregnancy or among pediatrics, or 4) studies evaluating therapeutic intervention which includes DKA secondary to sodium-glucose co-transporter 2 inhibitor.

\section{Outcomes of interest}

\section{Primary outcome}

Level of serum potassium, and $\mathrm{pH}$-adjusted corrected level of potassium at the time of admission.

\section{Secondary outcome}

Cardiovascular outcomes in relation to potassium in DKA episode

Specifically, the CV outcomes were noted in relation to the level of potassium at the time of occurrence of CV event and included ECG, report of fibrillation, tachycardia or bradycardia, central venous pressure, cardiac arrest, myocardial infarction, and troponin. Moreover, reports of CV outcomes or signs and symptoms were recorded if the CV outcome was reported as the reason for fatality. It was further noted if any relationship was given by the authors between hypokalemia and the reason for such $\mathrm{CV}$ outcome.

\section{Data extraction and synthesis}

All references retrieved were initially grouped under the respective search engine. Duplicates were removed, and titles were screened for eligibility. After removal of irrelevant studies, regrouping was done according to the nature of the study, ie, case series, clinical trial, guideline, etc. Relevant demographic, scientific, and clinical information was recorded in a separate data extraction sheet (Supplement). Later, the level of corrected potassium was calculated by subtracting $0.6 \mathrm{mmol} / \mathrm{L}$ from the reported potassium level against each 0.1 decreases in arterial $\mathrm{pH}$ from 7.4. ${ }^{15}$

Two of the authors (AU and TMK) independently reviewed the titles and abstracts of all identified studies to determine the articles that were suitable for further consideration. Disagreements were resolved by discussion among AU and TMK. A standardized form was used to extract the data from selected studies which included authors, year of publication, country of conduct of the study, region of the country, study objectives, sample size, the duration for study, study design and nature, and level of reported potassium and $\mathrm{pH}$ (Supplement). The extracted data was subsequently reviewed independently by IR and $A B$, whereas verification of extraction was done by TMK, NM, and MMB. Disagreements encountered in data synthesis, if any, were resolved by discussion between AU and TMK, and $\mathrm{IR}$ and $\mathrm{AB}$. The value of the $\mathrm{pH}$-adjusted corrected potassium level was later added to the respective study.

\section{Quality assessment}

The quality of randomized clinical trials was assessed using the Cochrane risk of bias tool, ${ }^{28}$ while observational studies were assessed by using the Newcastle Ottawa Scale for cross-sectional and cohort studies. ${ }^{29,30}$ For Cochrane risk of bias, bias that was likely to affect the results seriously was graded as high risk, bias that was unlikely to affect results seriously was graded as low risk, and bias that was likely to raise doubts regarding results was graded as unclear bias. ${ }^{28}$

The Newcastle Ottawa scale for cross-sectional studies was scored as: "selection (up to 4 points), comparability (up to 2 points), and outcome (up to 3 points)". ${ }^{29}$ A similar standard was used for the Newcastle Ottawa scale for cohort studies as 
well. ${ }^{30}$ Overall, the study quality was labeled as poor (score $\leq 3$ ), fair (score 4-6), and good (score $\geq 7$ ). Quality assessment was solely done by AU and independently reviewed by IR, AB, and TMK. Lastly, TMK and SLWH resolved disagreements from quality assessment through discussion.

\section{Results}

\section{Study selection}

The search identified 5,083 studies after removing duplicates $(5,229)$ and title screening $(2,968)$ (Figure 1). Of these, 2,115 studies were selected for abstract screening. A total of 2,039 studies were excluded as they did not have relevant data related to DKA (502), were reviews (440), were published in a language other than English (205), or either had a patient pool of pregnant or pediatric patients (228). The review is concluded on 47 studies. ${ }^{9,16,21,31-74}$

\section{Characteristics of included full-text retrievals}

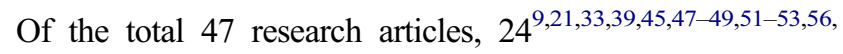
58-60,62-64,68-71,73,74 were cross-sectional case series mainly describing retrospective data on either prevalence or simple characteristics of DKA patients upon admission (Table 1). Two of the studies ${ }^{38,65}$ were cohorts of DKA patients further divided on the basis of treatment intervention given at a given time and retrospectively evaluated for the efficacy or safety of the said treatment intervention. Seven studies ${ }^{37,40,41,43,55,57,67}$ were clinical trials that prospectively measured such effects; however, only five of these studies ${ }^{40,43,55,57,67}$ were randomized control trials. Only 37 of the studies concurrently reported the value of $\mathrm{pH}$ and potassium, ${ }^{16,34,35,37,40-44,46-}$ 50,52-74 six provided only CV outcomes, ${ }^{9,31-33,36,45}$ and only four ${ }^{21,38,39,51}$ discussed potassium, $\mathrm{pH}$, and $\mathrm{CV}$ outcomes concurrently (Table 1).

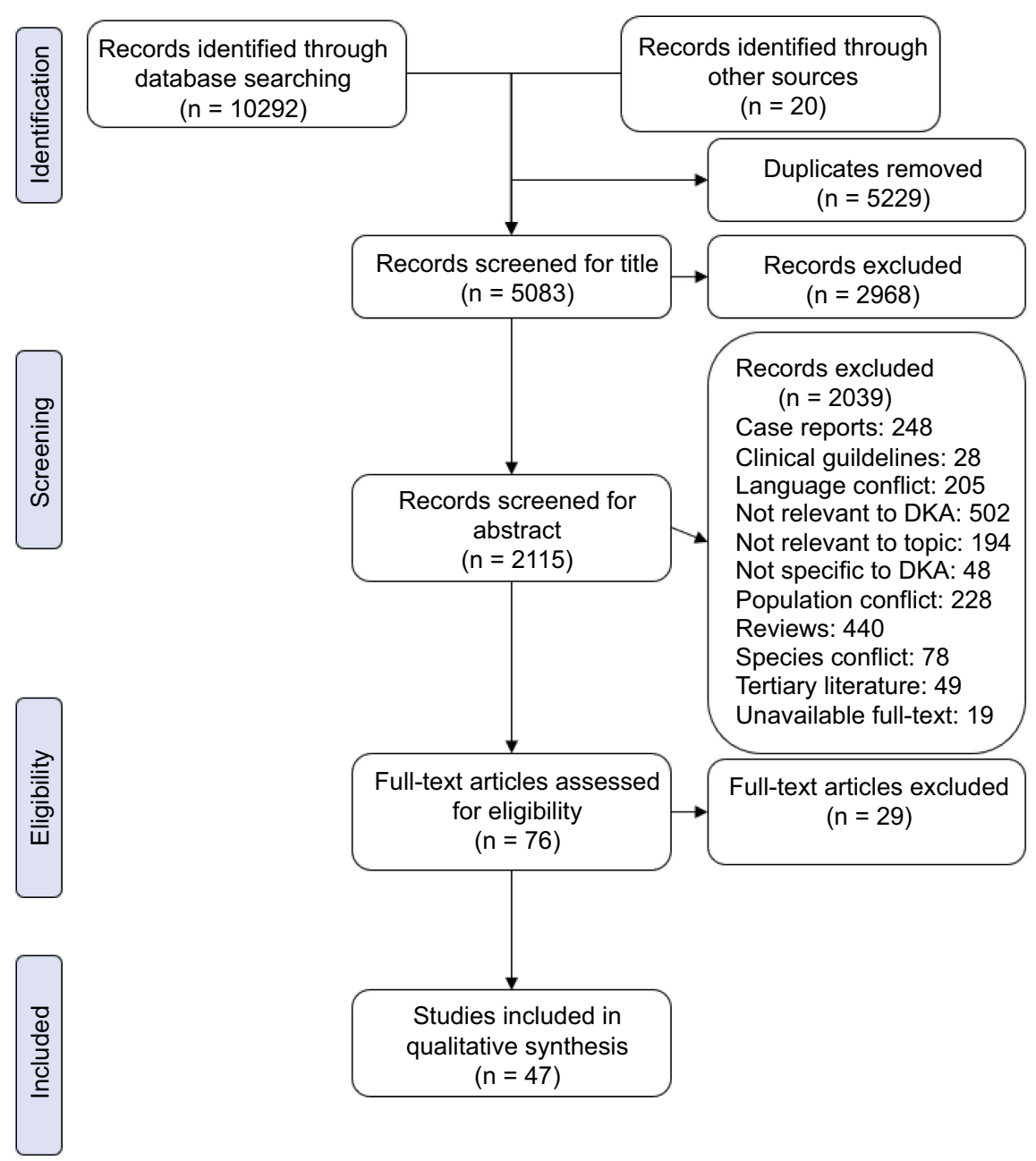

Figure I PRISMA flow chart of inclusion and exclusion of studies in systematic review. Note: Adapted from PRISMA. ${ }^{25}$

Abbreviation: DKA, diabetic ketoacidosis. 


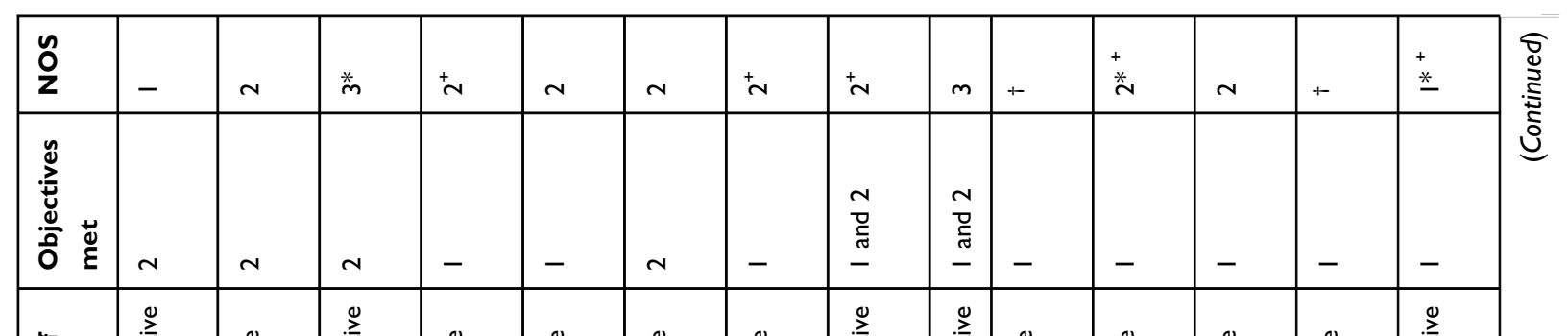

\begin{tabular}{|c|c|c|c|c|c|c|c|c|c|c|c|c|c|c|}
\hline 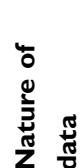 & 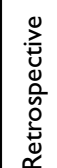 & 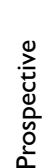 & 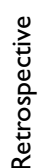 & 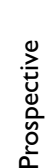 & 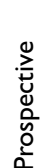 & 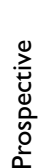 & 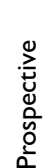 & 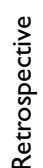 & 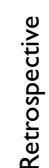 & 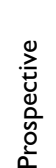 & 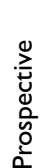 & 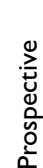 & 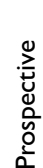 & 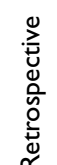 \\
\hline
\end{tabular}

\begin{tabular}{|c|c|c|c|c|c|c|c|c|c|c|c|c|c|}
\hline 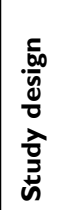 & 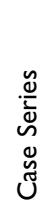 & 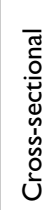 & 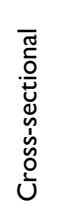 & $\begin{array}{l}\frac{5}{0} \\
\text { o } \\
\text { D. }\end{array}$ & 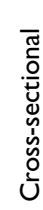 & 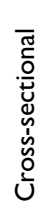 & $\begin{array}{l}\frac{1}{0} \\
\frac{0}{0} \\
0 \\
0 \\
0 \\
.0 \\
.0 \\
0 \\
0\end{array}$ & $\begin{array}{l}\frac{1}{0} \\
\text { Oे }\end{array}$ & 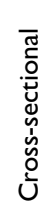 & 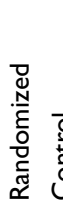 & 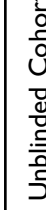 & 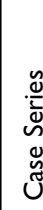 & 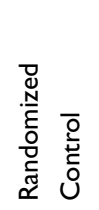 \\
\hline
\end{tabular}

\begin{tabular}{|c|c|c|c|c|c|c|c|c|c|c|c|c|c|c|}
\hline 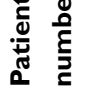 & $\stackrel{\circ}{\circ}$ & m & 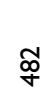 & $\sigma$ & $=$ & $\bar{m}$ & $\underline{m}$ & $\stackrel{\infty}{n}$ & $\pi$ & $\stackrel{\infty}{+}$ & $\stackrel{ \pm}{ \pm}$ & 0 & $\stackrel{m}{~}$ & 으 \\
\hline & $\grave{\Phi}$ & & $\frac{n}{b}$ & & & $\frac{\overline{0}}{n}$ & $\geq_{0,0}^{\geq}$ & & & $\subseteq$ & 0 & $\begin{array}{l}\stackrel{0}{\circ} \\
\stackrel{0}{0}\end{array}$ & ' & \\
\hline
\end{tabular}

\begin{tabular}{|c|c|c|c|c|c|c|c|c|c|c|c|c|c|c|}
\hline 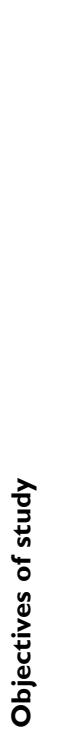 & 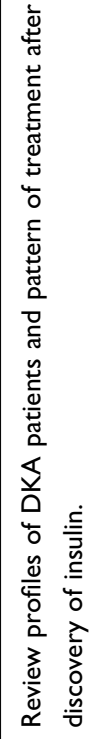 & 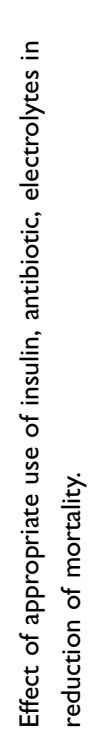 & 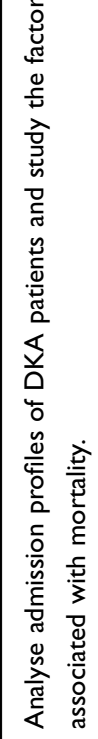 & 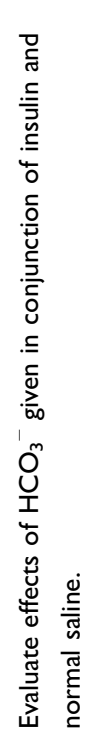 & 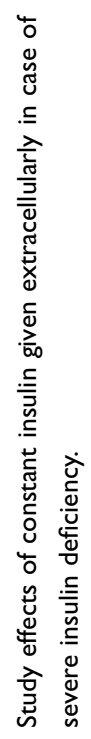 & 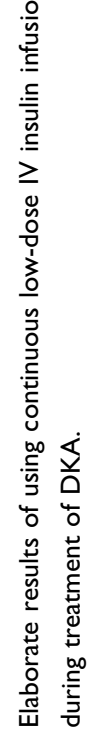 & 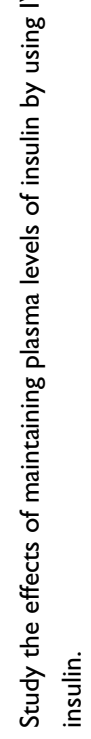 & 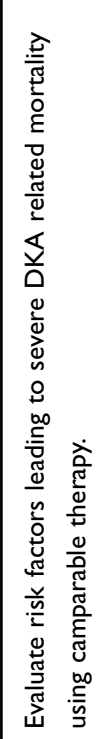 & 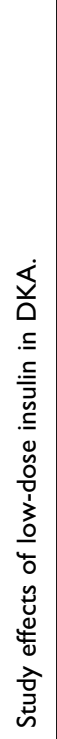 & 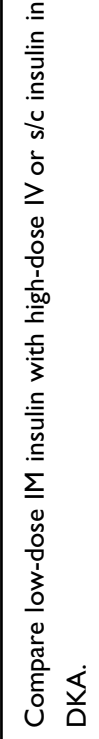 & 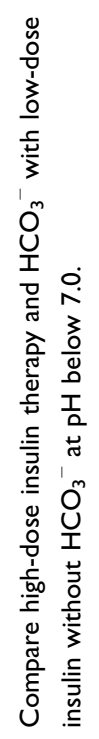 & 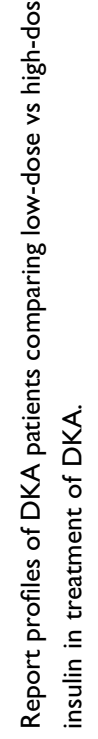 & 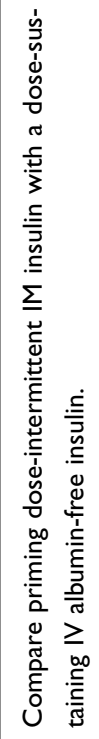 & 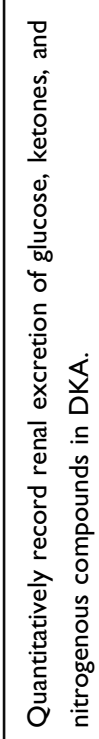 \\
\hline 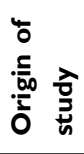 & 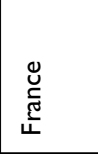 & 刹 & 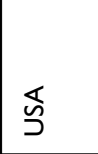 & 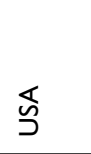 & 芯 & 弚 & 弚 & 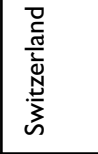 & 弚 & 芯 & 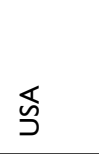 & 吕 & 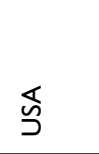 & 岁 \\
\hline 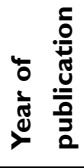 & $\stackrel{\varrho}{\underline{\varrho}}$ & 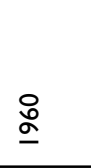 & $\underline{\underline{\Omega}}$ & 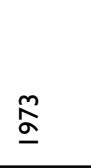 & 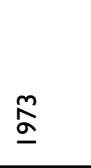 & $\underset{\sigma}{\stackrel{+}{\sigma}}$ & $\underset{⿱ 亠}{\alpha}$ & 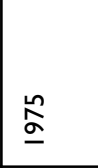 & 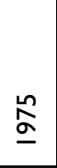 & $\stackrel{2}{2}$ & $\underset{\alpha}{\alpha}$ & $\underset{\Omega}{\alpha}$ & $\frac{\Omega}{\alpha}$ & $\stackrel{\bar{\alpha}}{\stackrel{\alpha}{\alpha}}$ \\
\hline $\begin{array}{l}\frac{n}{0} \\
\frac{\dot{q}}{y} \\
\frac{y}{4}\end{array}$ & 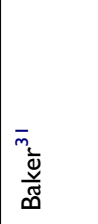 & 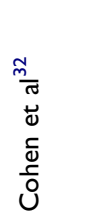 & 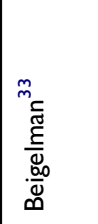 & 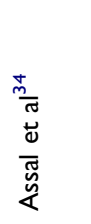 & 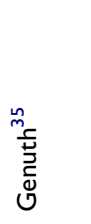 & 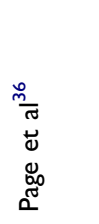 & 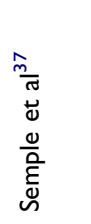 & 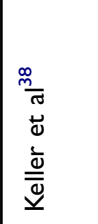 & 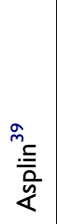 & 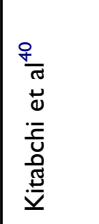 & 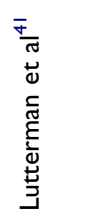 & 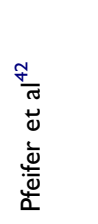 & $\begin{array}{l}\frac{\tilde{q}}{\pi} \\
\tilde{0} \\
\tilde{y} \\
\tilde{y} \\
\tilde{n}\end{array}$ & 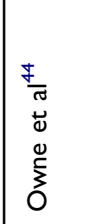 \\
\hline
\end{tabular}




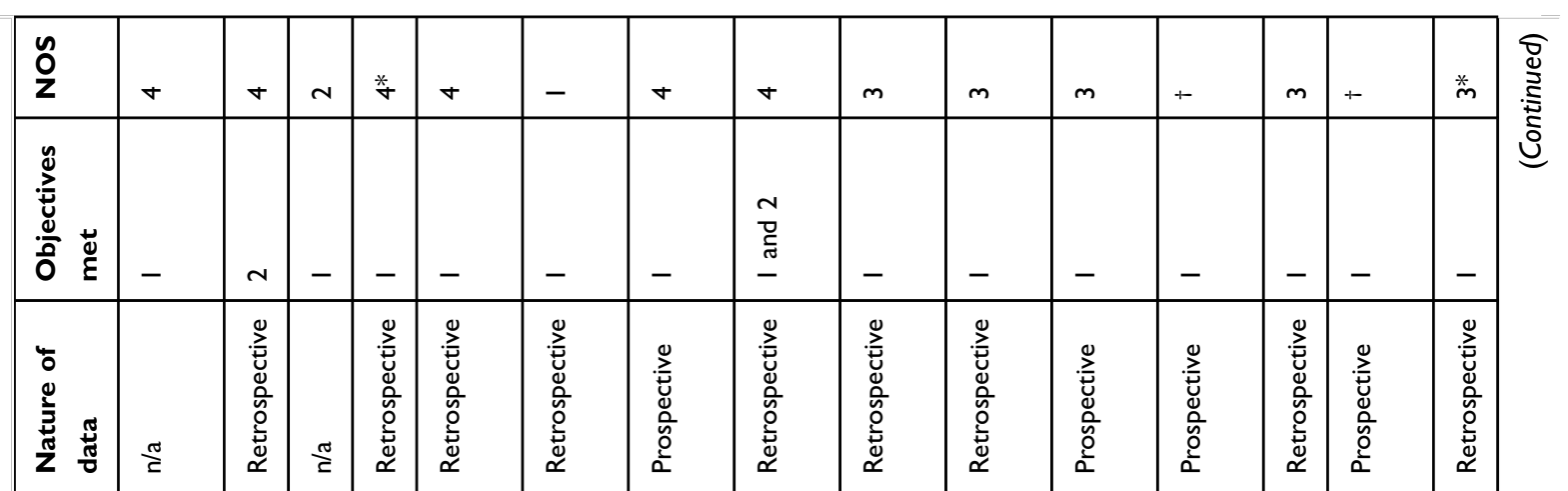

\begin{tabular}{|c|c|c|c|c|c|c|c|c|c|c|c|c|c|c|c|}
\hline 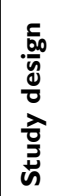 & 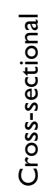 & 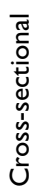 & 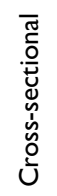 & 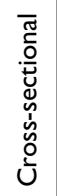 & 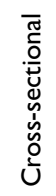 & 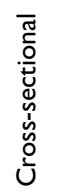 & 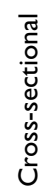 & 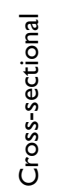 & 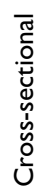 & 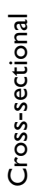 & 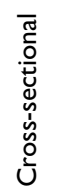 & 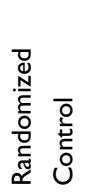 & 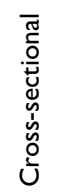 & 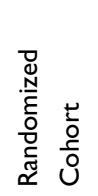 & 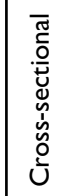 \\
\hline
\end{tabular}

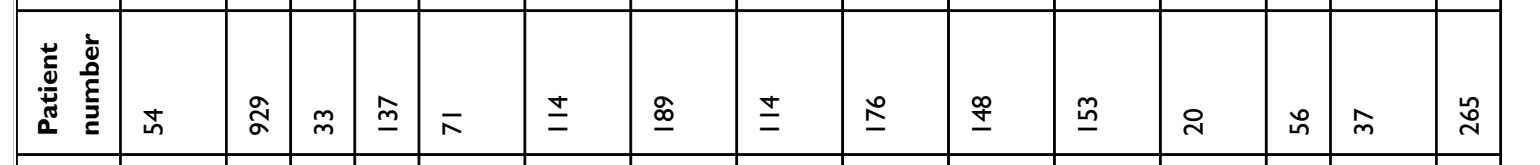

\begin{tabular}{|c|c|c|c|c|c|c|c|c|c|c|c|c|c|c|c|}
\hline 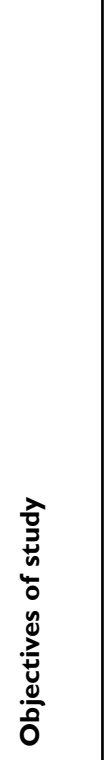 & 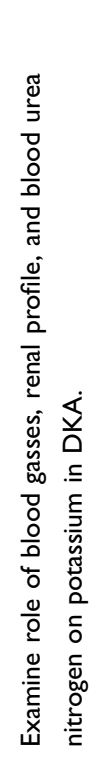 & 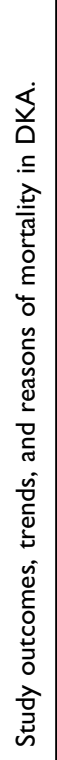 & 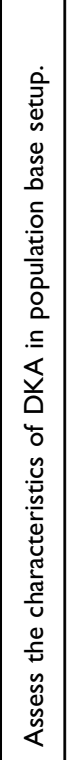 & 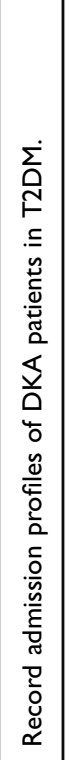 & 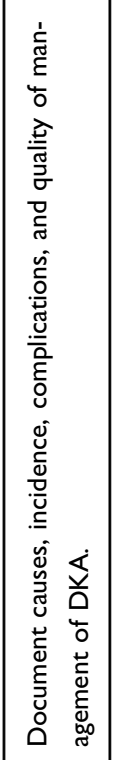 & 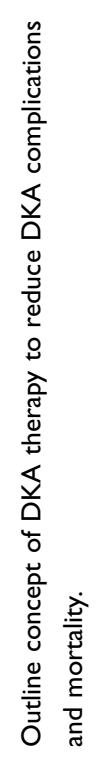 & 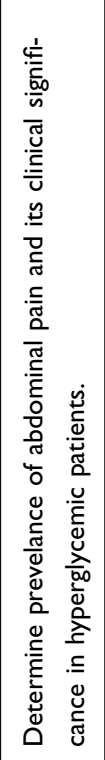 & 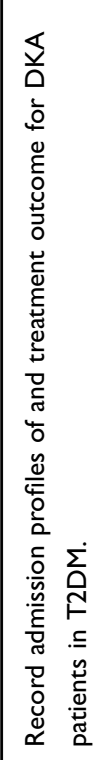 & 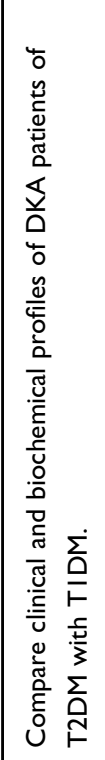 & 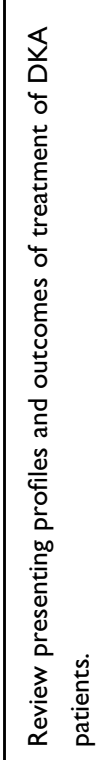 & 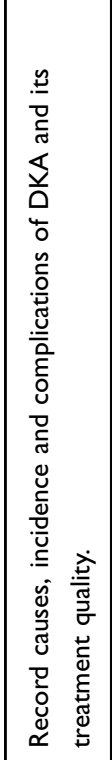 & 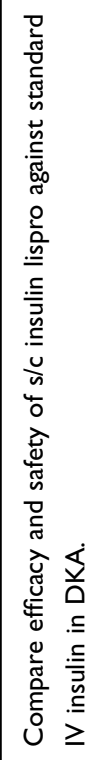 & 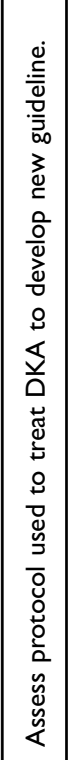 & 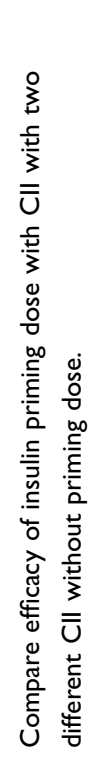 & 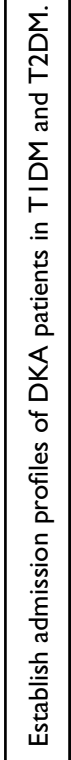 \\
\hline 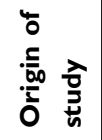 & 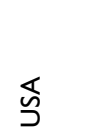 & 弚 & 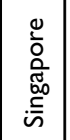 & 谞 & 弚 & 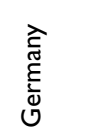 & 吕 & 畕 & 吕 & 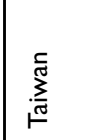 & 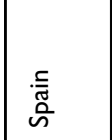 & 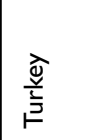 & $\frac{\frac{\pi}{\pi}}{\sum}$ & 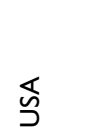 & 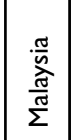 \\
\hline 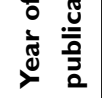 & $\stackrel{\circ}{\circ}$ & $\sigma$ & $\stackrel{\tilde{\sigma}}{\alpha}$ & $\stackrel{\widehat{\alpha}}{\alpha}$ & $\stackrel{\alpha}{\alpha}$ & $\alpha$ & రి & ષ્તે & ర్రి & ڤ్రి & ઠัర & ఫ్రి & $\stackrel{\infty}{\stackrel{N}{\circ}}$ & ஜ्రి & ఫ్రి \\
\hline 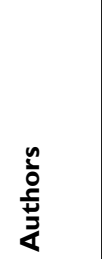 & 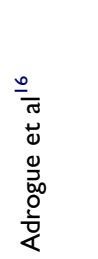 & 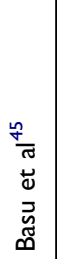 & 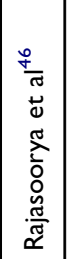 & 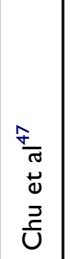 & 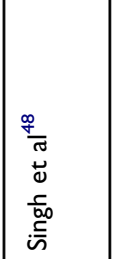 & 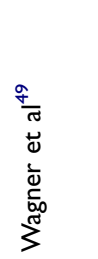 & 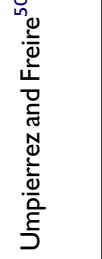 & 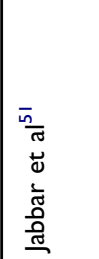 & 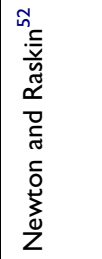 & 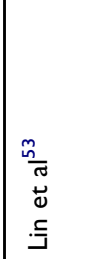 & 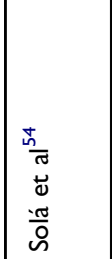 & 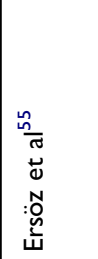 & $\begin{array}{l}\frac{0}{n} \\
\frac{0}{0} \\
\stackrel{0}{0} \\
\frac{\pi}{0} \\
\frac{0}{\alpha}\end{array}$ & 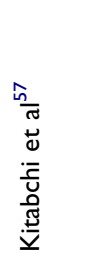 & 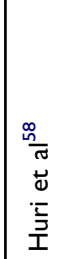 \\
\hline
\end{tabular}




\begin{tabular}{|c|c|c|c|c|c|c|c|c|c|c|c|c|c|c|c|}
\hline $\begin{array}{l}n \\
\text { o } \\
z\end{array}$ & $N$ & in & $\stackrel{*}{m}$ & 苫 & ᄂ & + & $\begin{array}{l}+ \\
\stackrel{*}{N}\end{array}$ & $\nabla$ & + & in & 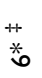 & L & Ln & $N$ & 0 \\
\hline 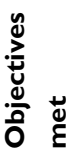 & - & - & - & - & - & - & - & - & - & - & - & - & $N$ & - & 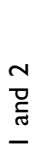 \\
\hline 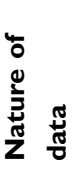 & 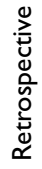 & 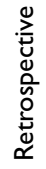 & 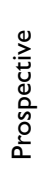 & 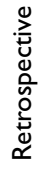 & 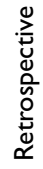 & 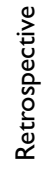 & 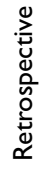 & 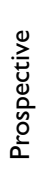 & 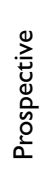 & 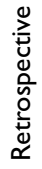 & 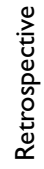 & 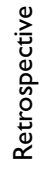 & 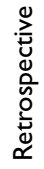 & 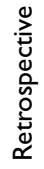 & 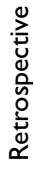 \\
\hline
\end{tabular}

\begin{tabular}{|c|c|c|c|c|c|c|c|c|c|c|c|c|c|c|c|}
\hline 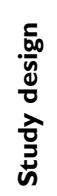 & 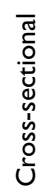 & 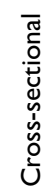 & 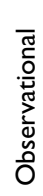 & 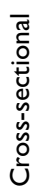 & 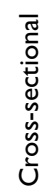 & 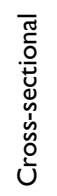 & 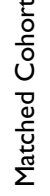 & 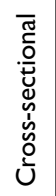 & 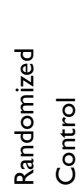 & 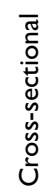 & 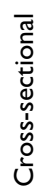 & 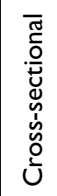 & 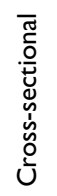 & 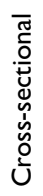 & 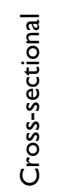 \\
\hline
\end{tabular}

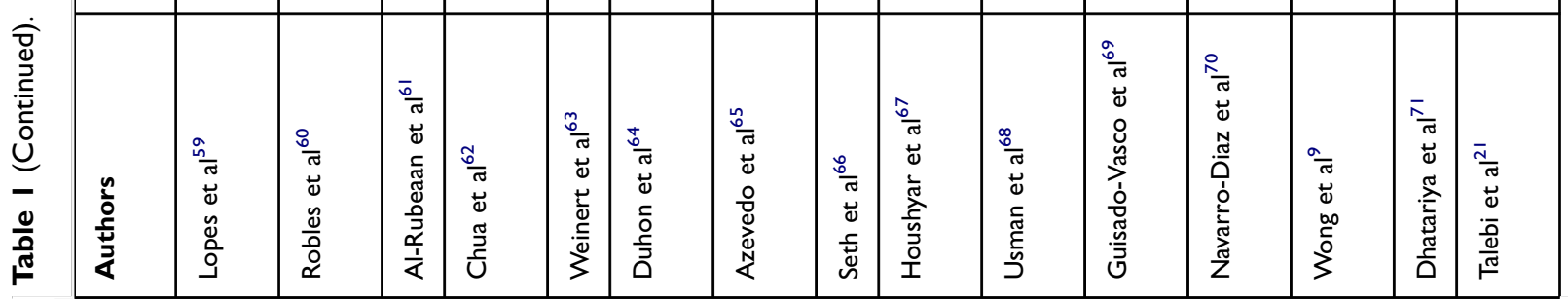




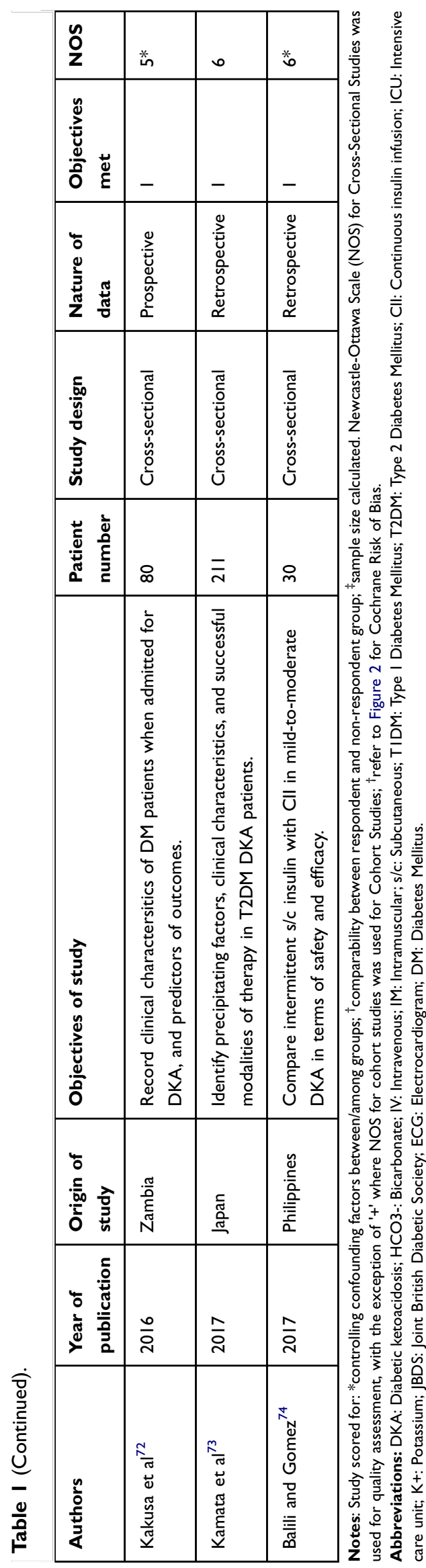

\section{Quality of the included studies}

In terms of quality of the studies, 22 studies $^{31-39,41,42,44,46,49,52-54,56,58,59,61,71}$ scored poor, 18 studies $^{9,16,21,45,47,48,50,51,60,62,63,66,68-70,72-74}$ scored fair, and two studies ${ }^{64,65}$ scored good on NOS. Among studies scoring 5 , four ${ }^{43,55,62,72}$ were scored on the basis of comparability by controlling confounding factors between the groups as well. Similarly, of the studies that scored 3 and 4 on NOS, three studies ${ }^{33,58,61}$ from score 3, and two studies ${ }^{47,57}$ from score 4 were also scored for controlling confounding factors between the groups (Table 1 footnote). Of four studies that established comparability between respondents and non-respondents, one each scored $4^{40}$ and $6^{57}$ on NOS and two scored 7. ${ }^{64,67}$ Notably, there was only one study that defined the sample size in terms of calculating on the basis of prevalence of DKA. ${ }^{69}$

The Cochrane Risk of Bias tool was used to assess five studies. ${ }^{40,43,55,57,67}$ None of the studies was completely blinded for participants (Figure 2). In the context of blinding of outcomes, only two of the studies ${ }^{55,67}$ predefined outcomes for assessment of endpoints. Among these studies, the least reporting bias was observed in the study by Ersöz et al, ${ }^{55}$ while the rest of the studies were found with a moderate risk of bias. ${ }^{40,43,57,67}$

\section{Data analysis: potassium, $\mathrm{pH}$, and $\mathrm{pH}$-adjusted corrected potassium}

Concurrently, the level of potassium and $\mathrm{pH}$ was only available in 41 studies $^{16,21,34,35,37-44,46-74}$ (Table 2). The least actual

\begin{tabular}{|c|c|c|c|}
\hline Study & Year & & \\
\hline Kitabchi [40] & 1976 & + & ? \\
\hline Sacks [43] & 1979 & + & ? \\
\hline Erasǒz [55] & 2006 & + & + \\
\hline Kitabchi [57] & 2008 & + & + \\
\hline Houshyar [67] & 2015 & $?$ & + \\
\hline
\end{tabular}

Figure 2 Cochrane Risk of Bias for included clinical trials. 
Table 2 Measure and $\mathrm{pH}$ adjusted potassium level in the included studies grouped on the basis of region

\begin{tabular}{|c|c|c|c|c|c|c|c|c|}
\hline Authors & Region & $\begin{array}{l}\text { Patient } \\
\text { number }\end{array}$ & $\begin{array}{l}\text { Reported } \\
\mathrm{K}^{+} \text {level } \\
\text { mean } \\
(\mathrm{SD} / \mathrm{QQR})\end{array}$ & $\begin{array}{l}\text { Region- } \\
\text { wise } \mathrm{K}^{+} \\
\text {level } \\
\mathrm{mmol} / \mathrm{L}\end{array}$ & $\begin{array}{l}\text { Admission } \\
\mathrm{pH} \text { mean } \\
\text { (SD/IQR) }\end{array}$ & $\begin{array}{l}\text { Region- } \\
\text { wise pH } \\
\text { level }\end{array}$ & $\begin{array}{l}\text { Calculated } \\
\text { potassium }\end{array}$ & $\begin{array}{l}\text { Regional } \\
\text { calculated } \\
\mathrm{K}^{+} \text {mmol/ } \\
\mathbf{L}\end{array}$ \\
\hline Rajasoorya et al ${ }^{46}$ & Far Eastern & \multirow[t]{8}{*}{979} & $5.1(I . I)$ & \multirow[t]{8}{*}{$4.9(0.44)$} & $7.15(0.16)$ & \multirow[t]{8}{*}{$7.15(0.065)$} & 3.9 & \multirow[t]{8}{*}{$3.65(0.38)$} \\
\hline Chu et $\mathrm{al}^{47}$ & Far Eastern & & $4.78(1.0)$ & & $7.14(0.25)$ & & 3.52 & \\
\hline Lin et $a^{53}$ & Far Eastern & & $4.6(1.0)$ & & $7.13(0.11)$ & & 3.28 & \\
\hline Huri et $\mathrm{al}^{58}$ & Far Eastern & & $5.22(1.43)$ & & $7.22(0.14)$ & & 4.22 & \\
\hline Chua et $\mathrm{al}^{62}$ & Far Eastern & & $5.5(3.5-7.3)$ & & $7.02(6.9-7.13)$ & & 3.52 & \\
\hline Usman et $\mathrm{al}^{68}$ & Far Eastern & & $4.3(1.2)$ & & $7.15(0.16)$ & & 3.1 & \\
\hline Kamata et $\mathrm{a}^{73}$ & Far Eastern & & $5.2(1.2)$ & & $7.16(0.14)$ & & 4.06 & \\
\hline Balili et $\mathrm{al}^{74}$ & Far Eastern & & $4.3(1.0)$ & & $7.24(0.13)$ & & 3.64 & \\
\hline Jabbar et $\mathrm{a}^{51}$ & Mid-Eastern & \multirow[t]{7}{*}{586} & $4.9(1.2)$ & \multirow[t]{7}{*}{$4.4(0.5 I)$} & $7.07(0.11)$ & \multirow[t]{7}{*}{$7.17(0.023)$} & 3.22 & \multirow[t]{7}{*}{$3.34(0.50)$} \\
\hline Ersöz et al ${ }^{55}$ & Mid-Eastern & & $4.8(0.7)$ & & $7.16(0.12)$ & & 3.76 & \\
\hline Abela et $\mathrm{al}^{56}$ & Mid-Eastern & & $3.4(n / a)$ & & $7.19(7.1-7.2)$ & & 2.4 & \\
\hline Al-Rubeaan et $\mathrm{al}^{61}$ & Mid-Eastern & & $4.5(0.8)$ & & $7.20(0.10)$ & & 3.6 & \\
\hline Seth et $\mathrm{al}^{66}$ & Mid-Eastern & & $4.5(n / a)$ & & $7.23(\mathrm{n} / \mathrm{a})$ & & 3.83 & \\
\hline Houshyar et al ${ }^{67}$ & Mid-Eastern & & $4.6(0.6)$ & & $7.09(0.15)$ & & 3.04 & \\
\hline Kakusa et $\mathrm{al}^{72}$ & Mid-Eastern & & $4.1(1.8)$ & & $7.26(0.15)$ & & 3.56 & \\
\hline Semple et $\mathrm{al}^{37}$ & Mid-Western & \multirow[t]{9}{*}{1995} & $5.3(1.1)$ & \multirow[t]{9}{*}{$4.9(0.3 \mathrm{I})$} & $7.07(0.18)$ & \multirow[t]{9}{*}{$7.12(0.058)$} & 3.62 & \multirow[t]{9}{*}{$3.51(0.28)$} \\
\hline Keller et $\mathrm{a}^{38}$ & Mid-Western & & $5.0(1.3)$ & & $7.00(0.20)$ & & 2.9 & \\
\hline Asplin 39 & Mid-Western & & $4.2(1.0)$ & & $7.19(0.13)$ & & 3.24 & \\
\hline Singh et al ${ }^{48}$ & Mid-Western & & $5.0(3.5-8.0)$ & & $7.16(6.57-7.32)$ & & 3.86 & \\
\hline Wagner et $\mathrm{al}^{49}$ & Mid-Western & & $5.1(0.9)$ & & $7.13(0.13)$ & & 3.78 & \\
\hline Solá et $\mathrm{al}^{54}$ & Mid-Western & & $4.9(0.96)$ & & $7.12(0.12)$ & & 3.52 & \\
\hline Dhatariya et al ${ }^{71}$ & Mid-Western & & $4.8(1.0)$ & & $7.16(0.15)$ & & 3.6 & \\
\hline Guisado-Vasco et al ${ }^{69}$ & Mid-Western & & $4.76(0.98)$ & & $7.16(0.19)$ & & 3.62 & \\
\hline Navarro-Diaz et al ${ }^{70}$ & Mid-Western & & $5.0(3.3-8.0)$ & & $7.10(6.4-7.3)$ & & 3.5 & \\
\hline Assal et $\mathrm{al}^{34}$ & Far Western & \multirow[t]{8}{*}{1554} & $5.6(0.3)$ & \multirow[t]{8}{*}{$5.1(0.45)$} & $7.06(0.03)$ & \multirow[t]{8}{*}{$7.11(0.052)$} & 3.86 & \multirow[t]{8}{*}{$3.62(0.52)$} \\
\hline Genuth $^{35}$ & Far Western & & $5.1(0.8)$ & & $7.12(0.12)$ & & 3.72 & \\
\hline Kitabchi et $\mathrm{al}^{40}$ & Far Western & & $5.6(0.25)$ & & $7.14(0.02)$ & & 4.34 & \\
\hline Lutterman et al ${ }^{4}$ & Far Western & & $5.3(1.75)$ & & $6.93(0.09)$ & & 2.78 & \\
\hline Pfeifer et $\mathrm{al}^{42}$ & Far Western & & $5.1(1.0)$ & & $7.00(0.12)$ & & 3 & \\
\hline Sacks et $\mathrm{al}^{43}$ & Far Western & & $5.8(0.3)$ & & $7.08(0.05)$ & & 4.18 & \\
\hline Owne et $\mathrm{al}^{44}$ & Far Western & & $4.2(0.2)$ & & $7.28(0.02)$ & & 3.78 & \\
\hline Adrogue et $\mathrm{al}^{16}$ & Far Western & & $5.7(I . I)$ & & $7.06(0.11)$ & & 3.96 & \\
\hline
\end{tabular}

(Continued) 
Table 2 (Continued).

\begin{tabular}{|c|c|c|c|c|c|c|c|c|}
\hline Authors & Region & $\begin{array}{l}\text { Patient } \\
\text { number }\end{array}$ & $\begin{array}{l}\text { Reported } \\
\mathrm{K}^{+} \text {level } \\
\text { mean } \\
\text { (SD/IQR) }\end{array}$ & $\begin{array}{l}\text { Region- } \\
\text { wise } \mathrm{K}^{+} \\
\text {level } \\
\mathrm{mmol} / \mathrm{L}\end{array}$ & $\begin{array}{l}\text { Admission } \\
\text { pH mean } \\
\text { (SD/IQR) }\end{array}$ & $\begin{array}{l}\text { Region- } \\
\text { wise pH } \\
\text { level }\end{array}$ & $\begin{array}{l}\text { Calculated } \\
\text { potassium }\end{array}$ & $\begin{array}{l}\text { Regional } \\
\text { calculated } \\
\mathbf{K}^{+} \text {mmol/ } \\
\mathbf{L}\end{array}$ \\
\hline $\begin{array}{l}\text { Umpierrez and } \\
\text { Freire }^{50}\end{array}$ & Far Western & & $5.4(0.5)$ & & $7.12(0.05)$ & & 4.02 & \\
\hline Newton and Raskin 52 & Far Western & & $4.7(0.7)$ & & $7.23(0.09)$ & & 3.98 & \\
\hline Kitabchi ${ }^{57}$ & Far Western & & $5.2(0.25)$ & & $7.11(0.03)$ & & 3.76 & \\
\hline Lopes $^{59}$ & Far Western & & $4.7(1.6)$ & & $7.17(0.18)$ & & 3.62 & \\
\hline Robles $^{60}$ & Far Western & & $4.2(0.9)$ & & $7.11(0.15)$ & & 2.76 & \\
\hline Weinert ${ }^{63}$ & Far Western & & $4.9(0.7)$ & & $7.17(0.12)$ & & 3.82 & \\
\hline Duhon $^{64}$ & Far Western & & $4.95(n / a)$ & & $6.96(n / a)$ & & 2.6 & \\
\hline Azevedo ${ }^{65}$ & Far Western & & $4.9(1.5)$ & & $7.11(0.15)$ & & 3.46 & \\
\hline Talebi $^{21}$ & Far Western & & $5.0(\mathrm{n} / \mathrm{a})$ & & $7.19(n / a)$ & & 4.04 & \\
\hline
\end{tabular}

Abbreviation: $\mathrm{K}+$, potassium.

value of potassium was noted at $3.4 \mathrm{mmol} / \mathrm{L},{ }^{56}$ while the maximum was $5.8 \mathrm{mmol} / \mathrm{L}^{43}$ On average, the potassium level was $5.8 \mathrm{mmol} / \mathrm{L}$. In a similar context, the least observed $\mathrm{pH}$ was $6.93^{41}$ and the highest $7.28,{ }^{44}$ with an average $\mathrm{pH}$ being 7.13. The level of potassium after adjusting against $\mathrm{pH}$ ranged from 2.4-4.34 (Table 2), with an average of 3.56 $\mathrm{mmol} / \mathrm{L}$. The corresponding values for actual potassium level and $\mathrm{pH}$ of mentioned values was $3.4 \mathrm{mmol} / \mathrm{L}$ with a $\mathrm{pH}$ of $7.19,{ }^{56}$ and 5.6 with a $\mathrm{pH}$ of $7.14 .^{40}$ Thirteen included studies $^{38,39,41,42,51,53,60,64,65,67,68,70}$ calculated the value of potassium to be equal or below $3.5 \mathrm{mmol} / \mathrm{L}$ when the patients sought medical advice; out of these, only one study ${ }^{56}$ reported the average actual potassium level to be lower than $3.5 \mathrm{mmol} /$ L. Upon distributing the countries on the basis of their region, it was notable that the least potassium level was seen in midEastern countries $(4.4 \mathrm{mmol} / \mathrm{L})$; the minimum $\mathrm{pH}$ was observed in the far-Western region (7.11); and the maximum number of patient data originated from mid-Western countries (patient number $=1,995$ ). Nevertheless, a meta-analysis was not performed as quantitative data was not sufficient for further analysis.

\section{Cardiovascular outcomes}

Of a total of 10 studies $9,31-33,36,38,39,45,51$ discussing CV outcomes, only four ${ }^{21,38,39,51}$ reported potassium and $\mathrm{pH}$, and discussed CV outcomes concurrently (Table 3). Two ${ }^{31,45}$ of the six remaining studies neither provided $\mathrm{pH}$ nor potassium level in the report. The maximum number of patients reported with CV disorder in any single study was 17 , where abnormal ECG was mentioned with recorded CV abnormality. ${ }^{32}$ In the context of insulin, one death was reported secondary to an increase in insulin. ${ }^{36}$ Interestingly, one study ${ }^{9}$ evaluated the extent of hypokalemia in DKA and reported three deaths to myocardial infarction (MI); however, none of the deaths were attributed to hypokalemia. Only one study ${ }^{33}$ mentioned the difference between the potassium level of alive and deceased DKA patients.

\section{Discussion}

This is the first systematic review which aims to explore $\mathrm{CV}$ events in patients of DKA in correlation with admission serum potassium vs $\mathrm{pH}$-adjusted corrected potassium level. Moreover, it is also an attempt to signify the importance of $\mathrm{pH}$-adjusted corrected potassium level in the diagnosis and management of DKA episode when weighed against the risk of $\mathrm{CV}$ events during an episode. In this regard, 45 studies reported admission potassium level of DKA patients and the $\mathrm{pH}$-adjusted corrected potassium level was calculable for 41 studies only. However, none of the studies reported a $\mathrm{pH}$-adjusted corrected potassium level and, hence, it was self-calculated to find the difference between the two values. Similarly, as for secondary outcome, 10 studies focused on the secondary outcome of a CV event in DKA, while none of the studies correlated $\mathrm{CV}$ outcomes with either reported or $\mathrm{pH}$ adjusted corrected potassium level. 


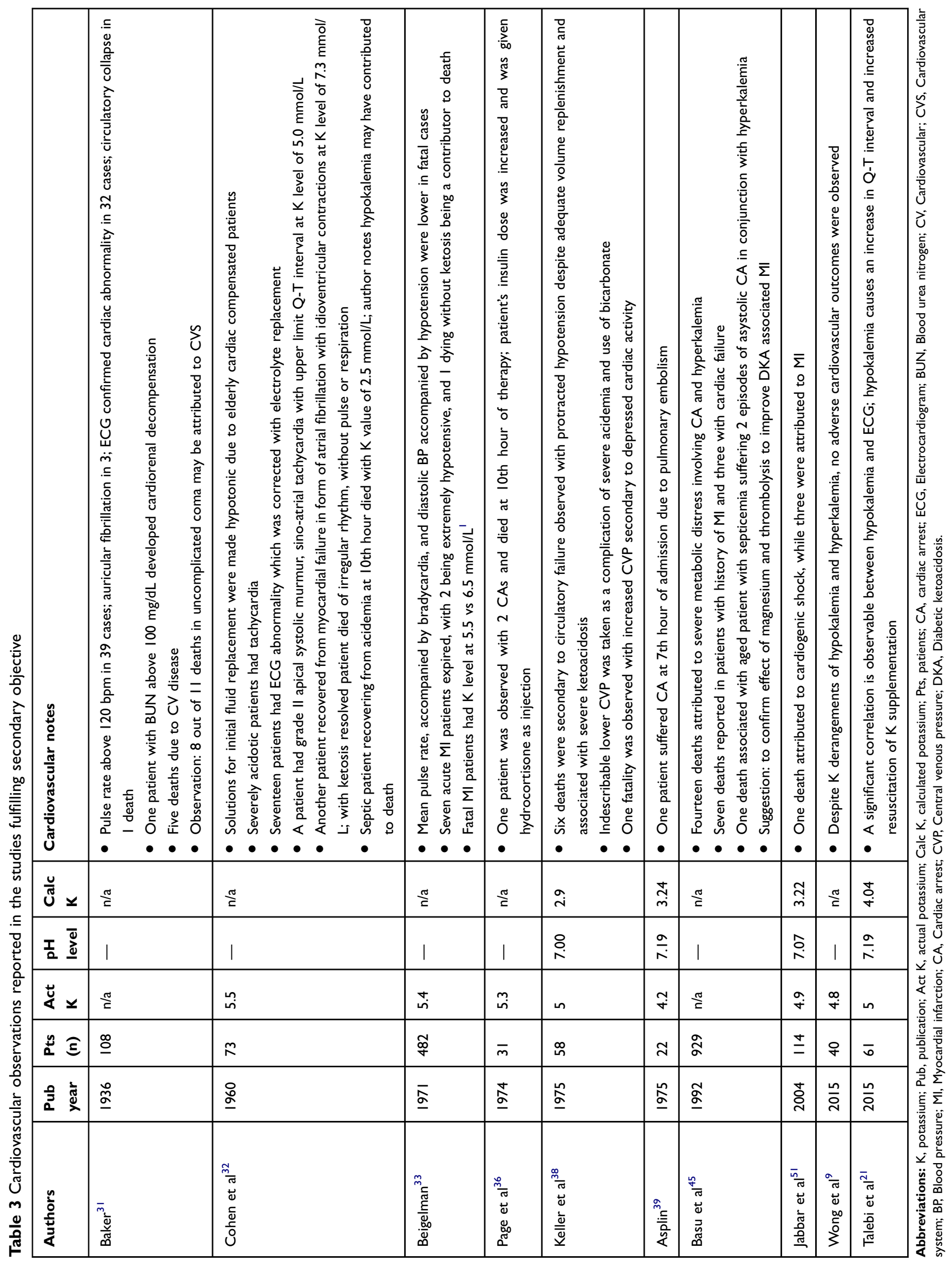


The reported admission potassium level in current review was $4.91 \mathrm{mmol} / \mathrm{L}$. Contrary to significant loss of potassium, DKA patients usually present with normal or increased potassium level, which is an established phenomenon and, hence, the observed value seems to be well within the normal range. ${ }^{9,15}$ Moreover, most of the clinical guidelines do not suggest that potassium supplementation shall be started once the value of serum potassium is below $4.5 \mathrm{mmol} / \mathrm{L}^{.4-7}$ Similarly, the mean $\mathrm{pH}$-adjusted corrected potassium level observed in the current review was within the normal range of potassium level as well $(3.56 \mathrm{mmol} / \mathrm{L})$, although this value is borderline-low. However, such normo- or hyperkalemia was deceiving in nature as there were 13 studies $^{38,39,41,42,51,53,56,60,64,65,67,68,70}$ which had a corrected potassium level below $3.5 \mathrm{mmol} / \mathrm{L}$. Further findings of the current review were in line with an aforementioned hypothesis where, in five of the studies, ${ }^{38,41,56,60,64} \mathrm{pH}$-adjusted corrected potassium was as low to produce an adverse CV outcome resultant of hypokalemia. ${ }^{11,15}$ When these studies were analyzed for any reported $\mathrm{CV}$ event in correlation of potassium, only three of the studies ${ }^{38,39,51}$ reported such events; the results were, however, not in specificity to hypokalemia. The incidence of hypokalemia was very frequent in the study reported by Keller et al, ${ }^{38}$ where $23 \%$ of DKA cases experienced a low potassium level. However, it is not mentioned whether the extent of hypokalemic episodes was associated with any of the four cases of early $\mathrm{CV}$ adverse outcomes or not; the authors also confirmed that these fatalities were established cases of chronic CV disease. In another study with below-normal corrected potassium level, Asplin et al did not provide any hint to the extent of hypokalemia. ${ }^{39}$ Death was attributed to cardiac arrest 3 hours post-admission due to pulmonary embolism; however, the laboratory data of this patient seem insignificant in correlation to $\mathrm{pH}$-adjusted corrected potassium. Similarly, the mean level of corrected potassium was calculated to be $3.2 \mathrm{mmol} / \mathrm{L}$ in the study by Jabbar et al. ${ }^{51}$ Strikingly, the mortality rate was $21 \%$, out of which five deaths were attributed to myocardial infarction (MI). No further description of post-mortem reports was provided and, hence, the link of potassium with these events remains unknown. Beside the findings above, none of the studies reported any findings related to the $\mathrm{pH}$-adjusted corrected potassium level.

None of the studies reported any findings related to the $\mathrm{pH}$-adjusted corrected potassium level and only one reported $\mathrm{CV}$ outcomes of DKA with respect to potassium. ${ }^{32}$ It is understandable that an initial report that focused on clerking the files of DKA patients after the discovery of insulin did not focus on the potassium at all, despite adverse CV events being the reason for eight deaths in uncomplicated DKA. ${ }^{31}$ This is because it was Holler $^{8}$ who described the effect of insulin on potassium during the treatment of DKA, which later became a part of established protocols. The attempt by Cohen et $\mathrm{al}^{32}$ reported a detailed description of DKA admissions. The authors did report on the measured potassium level at the time of admission and also concluded for a case that hypokalemia might have been a reason for the death of a patient from septicemia. Nevertheless, any correlation between potassium level and $\mathrm{pH}$ was not used at the time of diagnosis.

Treatment of DKA consists of three modalities and all of these directly affect serum potassium level, ie, 1) volume resuscitation causes dilution of serum potassium concentration, 2) correction of hyperglycemia via insulin helps cellular re-entry of potassium, and 3) correction of acidosis utilizing bicarbonate causes renal clearance of potassium. ${ }^{4-7}$ Bicarbonate is a least recommended therapeutic intervention, and a recent systematic review in this regard also did not find benefits of bicarbonate in the resolution of DKA. ${ }^{75}$ Moreover, significance of potassium supplementation is much required crucial when bicarbonate is given with insulin. However, in the current review, there were four studies that evaluated bicarbonate in the therapy of DKA; one each by Assal et al, ${ }^{34}$ Duhon et al, ${ }^{64}$ Keller et al, ${ }^{38}$ and Lutterman et al. $^{41}$ Apart from the $\mathrm{pH}$-adjusted corrected potassium level for a study conducted by Assal et $\mathrm{al}^{34}(3.86 \mathrm{mmol} /$ L), the rest of the three studies had a very low level of corrected potassium and was calculated to be 2.9, 2.78, and $2.6 \mathrm{mmol} / \mathrm{L}$, respectively (Table 2). Such a low level of actual potassium put the question of interest on $\mathrm{CV}$ profiles of patients of these studies. Notably, only Keller et $\mathrm{al}^{38}$ reported an indescribable lower CVP which was translated as a complication of severe acidaemia and use of bicarbonate; both of these factors independently cause cardiac suppression and potassium excretion via diuresis. Neither any finding related to CV impact of DKA nor corrected potassium level has been reported in the rest of the studies. This finding of the current systematic review once again highlights the importance of adjustment of potassium with respect to acidosis so that the impact of serum potassium on $\mathrm{CV}$ outcomes can be dealt with in a way that further improves patient related outcomes of DKA patients. 
The extent of non-adherence to the treatment protocol for the management of DKA is also a reason for hypokalemic events. ${ }^{76}$ Abela et $\mathrm{al}^{56}$ reported that potassium was given to the patients at a later stage of DKA resolution, with the main reasons being lack of clear instructions or secondary to not following the DKA management protocol to analyze the blood for potassium concentration; importantly, the volume of potassium resuscitation was higher and aggressive when initiated. In a similar report, a study conducted by Dhatariya et $\mathrm{al}^{71}$ in the UK found that hypokalemia was observed in most of the patients. Additionally, non-adherence to the potassium management protocol was the highest component of deviation from treatment guidelines and observed in $20 \%$ of the cases. Moreover, as high as $67 \%$ of patients observed a value of potassium level below $4.0 \mathrm{mmol} / \mathrm{L}$ at least once during the course of hospitalization. The authors emphasized the fact that potassium replenishment, and not replacement, shall be done aggressively and insulin shall be used to maneuver the potassium level between 4.0-5.5 mmol/L. Singh et $\mathrm{al}^{48}$ also reported similar findings when they audited the implementation of DKA treatment guidelines in the UK. In the context of adherence with the potassium resuscitation, only $30 \%$ of the cases satisfied the recommended standard of administration of potassium chloride of 70 mmol. Furthermore, $31 \%$ of the cases were found to experience hypokalemic episode incidence which was even higher when compared with events of hypoglycemia (14\%). In terms of adherence to DKA treatment guidelines, nearly the same results were reported by Solá et $\mathrm{al}^{54}$ when only $20 \%$ of the cases satisfied the potassium resuscitation criterion which resulted in a high incidence of iatrogenic hypokalemia. In another study, Wong et $\mathrm{al}^{9}$ identify multiple entities during analyzing factors associated with non-adherence of physicians with guidelines and the event of hypokalemia. Among these were a failure to prevent, to monitor, to diagnose, and to treat hypokalemia appropriately. The study also reported that delay in initiating potassium resuscitation, inadequate amount of potassium supplementation, infrequent monitoring of potassium level, under-recognition on the hypokalemic episode, and failure to withhold insulin were the key errors which may cause an adverse event. Although the well established guidelines strongly give recommendations regarding potassium monitoring and supplementation in DKA, none of these guidelines suggest using the $\mathrm{pH}$-adjusted corrected potassium level to initiate potassium resuscitation or adjust the treatment plan. ${ }^{4-7}$ And on top of it, count to carry out the recommendations for potassium resuscitation remains very low, regardless of time, whether it is observed in the distant ${ }^{9,48,54,56,71}$ or near past. ${ }^{76}$ Even further, despite all the included studies in the current review that were either depicting important parameters of DKA or experimenting with its management to improve patient oriented outcomes, not a single study demonstrated the impact of potassium and acidosis on cardiovascular outcomes in DKA patients, which remains an area to explore further.

\section{Conclusion}

Potassium has an interesting outlook during the course of DKA. Despite being an intracellular ion and most affected entity throughout the development and resolution of DKA, we find that it is given the least consideration in the literature encompassing DKA. Given the extent of the current review, none of the studies in our cohort mentioned potassium in correlation with $\mathrm{pH}$. It is also worthy to look into under-utilization of $\mathrm{pH}$ to adjust and correct the level of potassium during diagnosis and management of DKA, despite DKA patients from almost $27 \%$ of the studies experienced hypokalemia. Furthermore, most of the clinical guidelines highlight the significance of potassium supplementation during the management of DKA. However, the current review also suggests that the implementation for such supplementation is not carried out effectively. This remains a strong recommendation for healthcare professionals given that the cardiovascular outcomes are not monitored in the context of potassium and acidosis. Moreover, the features of hypokalemia are extensively observable upon an incident; the reports do not encompass potassium, $\mathrm{pH}$ and $\mathrm{CV}$ disorders simultaneously, which remains an unexplored area for reputable diabetic associations worldwide.

\section{Strengths and limitations}

Current systematic review tends to include most of the relevant scientific literature, which also encompassed the literature considered weak in terms of authenticity, or being lower in the grading of scientific criteria; hence, the results, although genuine, may not reflect light on the exact extent of the problem. Moreover, more than one third of the literature included had low quality assessment score, further weakening the suggestion provided via this systematic review. In a similar context, unavailability of the quantitative data did not allow for meta-analysis where only one of the study fulfilled three objectives 
concurrently. Other than the literature quality, calculation of $\mathrm{pH}$ from venous and arterial blood may significantly be different for which most studies do not report the origin of sample. Similarly, although serum analysis is used mostly in a hospital setting to retrieve renal profile values, unless declared, it was not clear whether potassium was calculated using serum or plasma samples. Moreover, due to no funding of the project, the authors were not able to include non-English studies secondary to the cost of translation.

\section{Disclosure}

The authors report no conflicts of interest in regard to this work.

\section{References}

1. Kitabchi AE, Umpierrez GE, Murphy MB. Diabetic ketoacidosis and hyperosmolar state. In: DeFronzo RA, Ferrannini E, Zimmet P, Alberti KG, editors. International Textbook of Diabetes Mellitus. Fourth edition. Hoboken: John Wiley \& Sons, Ltd.; 2015:799-814. doi:10.1002/9781118387658.ch54.

2. Nyenwe EA, Kitabchi AE. The evolution of diabetic ketoacidosis: an update of its etiology, pathogenesis and management. Metabolism. 2016;65(4):507-521. doi:10.1016/j.metabol.2015.12.007

3. Lebovitz HE. Diabetic ketoacidosis. Lancet. 1995;345(8952):767772. doi:10.1016/s0140-6736(95)90645-2

4. Keller U. Diabetic ketoacidosis: current views on pathogenesis and treatment. Diabetologia. 1986;29(2):71-77.

5. National Collaborating Centre for Women's and Children's Health (UK). Diabetes (type 1 and type 2) in children and young people: diagnosis and management. London: National Institute for Health and Care Excellence (UK); 2015. Available from: https://www.ncbi.nlm. nih.gov/pubmed/26334077. Accessed: March 18, 2016.

6. Kitabchi AE, Umpierrez GE, Murphy MB, Kreisberg RA. Hyperglycemic crises in adult patients with diabetes: a consensus statement from the American Diabetes Association. Diabetes Care. 2006;29(12):2739-2748. doi:10.2337/dc06-9916

7. Savage MW, Dhatariya KK, Kilvert A, et al. Joint British Diabetes Societies guideline for the management of diabetic ketoacidosis. Diabetic Med. 2011;28(5):508-515. doi:10.1111/j.1464-5491.2011. 03246.x

8. Holler JW. Potassium deficiency occurring during the treatment of diabetic acidosis. J Am Med Assoc. 1946;131(15):1186-1189.

9. Wong B, Cheng A, Yu C, Goguen J. Examining the "Killer K" of diabetic ketoacidosis at a Tertiary Care Hospital: an exploratory study. Can J Diabetes. 2016;40(3):204-209. doi:10.1016/j.jcjd.2015. 10.002

10. Gennari FJ. Hypokalemia. N Engl J Med. 1998;339(7):451-458. doi:10.1056/NEJM199808133390707

11. Gandhi M, Suvarna TT. Cardiovascular complications in diabetic ketoacidosis. Int J Diab Dev Countries. 1995;15:132-133.

12. Kraut JA, Madias NE. Treatment of acute metabolic acidosis: a pathophysiologic approach. Nature Rev Nephrol. 2012;8(10):589601. doi:10.1038/nrneph.2012.186

13. Schaefer TJ, Wolford RW. Disorders of potassium. Emerg Med Clin North Am. 2005;23(3):723-747, viii-ix. doi:10.1016/j.emc.2005. 03.016

14. Chiasson JL, Aris-Jilwan N, Belanger R, et al. Diagnosis and treatment of diabetic ketoacidosis and the hyperglycemic hyperosmolar state. CMAJ. 2003;168(7):859-866.
15. Shargel L. Comprehensive Pharmacy Review for NAPLEX. Baltimore, MD: Wolters Kluwer Health/Lippincott Williams \& Wilkins; 2013.

16. Adrogue HJ, Lederer ED, Suki WN, Eknoyan G. Determinants of plasma potassium levels in diabetic ketoacidosis. Medicine (Baltimore). 1986;65(3):163-172. doi:10.1097/00005792-1986050 00-00004

17. IDF. Diabetes Atlas. International Diabetes Federation, 8th ed.; 2017. Available from: https://wwwidforg/component/attachments/ attachmentshtml?id=1405\&task=download. Accessed February 23, 2018

18. Umpierrez GE, Khajavi M, Kitabchi AE. Review: diabetic ketoacidosis and hyperglycemic hyperosmolar nonketotic syndrome. Am J Med Sci. 1996;311(5):225-233.

19. Sica DA, Struthers AD, Cushman WC, Wood M, Banas JS Jr., Epstein M. Importance of potassium in cardiovascular disease. $J$ Clin Hypertens (Greenwich). 2002;4(3):198-206.

20. Kjeldsen K. Hypokalemia and sudden cardiac death. Exp Clin Cardiol. 2010;15(4):e96-99.

21. Talebi S, Ghobadi F, Cacacho A, et al. Looking at diabetic ketoacidosis through electrocardiogram window! Am J Emerg Med. 2016;34 (2):263-265. doi:10.1016/j.ajem.2015.10.032

22. Soler NG, Bennett MA, Fitzgerald MG, Malins JM. Electrocardiogram as guide to potassium replacement in diabetic ketoacidosis. Diabetes. 1974;23(7):610-615. doi:10.2337/diab.23.7.610

23. Liamis G, Liberopoulos E, Barkas F, Elisaf M. Diabetes mellitus and electrolyte disorders. World J Clin Cases. 2014;2(10):488-496. doi:10.12998/wjcc.v2.i10.488

24. Abdo AS, Geraci SA. Significance of elevated cardiac troponin I in patients with diabetic ketoacidosis. J Miss State Med Assoc. 2013;54 (5):127-130.

25. Moher D, Liberati A, Tetzlaff J, Altman DG, Group P. Preferred reporting items for systematic reviews and meta-analyses: the PRISMA statement. Ann Intern Med. 2009;151(4):264-269, W264. doi:10.7326/0003-4819-151-4-200908180-00135

26. Andrade-Castellanos CA, Colunga-Lozano LE, Delgado-Figueroa N, Gonzalez-Padilla DA. Subcutaneous rapid-acting insulin analogues for diabetic ketoacidosis. Cochrane Database Syst Rev. 2016;1: CD011281.

27. Tran TTT, Pease A, Wood AJ, et al. Review of evidence for adult diabetic ketoacidosis management protocols. Front Endocrinol (Lausanne). 2017;8:106. doi:10.3389/fendo.2017.00106

28. Higgins JAD. Chapter 8: assessing risk of bias in included studies. Cochrane Handbook for Systematic Reviews of Interventions. 2008. Avaliable from: http://handbook.cochrane.org/chapter_8/8_assessing risk_of_bias_in_included_studies.htm. Accessed February 26, 2017.

29. Liu $\mathrm{Y}, \overline{\mathrm{He} \mathrm{Y}}, \mathrm{Li}$ T, et al. Risk of primary liver cancer associated with gallstones and cholecystectomy: a meta-analysis. PLoS One. 2014;9 (10):e109733. doi:10.1371/journal.pone.0109733

30. Wells G, Shea B, O'Connell D, et al. Newcastle-Ottawa quality assessment scale cohort studies. 2014. Available from: http://www. ohri.ca/programs/clinical_epidemiology/oxford.asp. Accessed January 21, 2018.

31. Baker TW. A clinical survey of one hundred and eight consecutive cases of diabetic coma. Arch Intern Med. 1936;58(3):373-406. doi:10.1001/archinte.1936.00170130002001

32. Cohen AS, Vance VK, Runyan JW Jr., Hurwitz D. Diabetic acidosis: an evaluation of the cause, course and therapy of 73 cases. Ann Intern Med. 1960;52:55-86. doi:10.7326/0003-4819-52-1-55

33. Beigelman PM. Severe diabetic ketoacidosis (diabetic "coma"). 482 episodes in 257 patients; experience of three years. Diabetes. 1971;20 (7):490-500. doi:10.2337/diab.20.7.490

34. Assal JP, Aoki TT, Manzano FM, Kozak GP. Metabolic effects of sodium bicarbonate in management of diabetic ketoacidosis. Diabetes. 1974;23(5):405-411. doi:10.2337/diab.23.5.405 
35. Genuth SM. Constant intravenous insulin infusion in diabetic ketoacidosis. Jama. 1973;223(12):1348-1351.

36. Page MM, Pyke DA, Watkins PJ, et al. Treatment of Diabetic Coma with Continuous Low-dose Infusion of Insulin. Br Med J. 1974;2 (5921):687-690. doi:10.1136/bmj.2.5921.687

37. Semple PF, White C, Manderson WG. Continuous intravenous infusion of small doses of insulin in treatment of diabetic ketoacidosis. $\mathrm{Br}$ Med J. 1974;2(5921):694-698. doi:10.1136/bmj.2.5921.694

38. Keller U, Berger W, Ritz R, Truog P. Course and prognosis of 86 episodes of diabetic coma. A five year experience with a uniform schedule of treatment. Diabetologia. 1975;11(2):93-100.

39. Asplin CM. Experience in a district general hospital using a low dose insulin regimen for diabetic coma. Bristol Med Chir J. 1976;91(339340):5-9.

40. Kitabchi AE, Ayyagari V, Guerra SM. The efficacy of low-dose versus conventional therapy of insulin for treatment of diabetic ketoacidosis. Ann Intern Med. 1976;84(6):633-638. doi:10.7326/0003-4819-84-6-633

41. Lutterman JA, Adriaansen AAJ, van 't Laar A. Treatment of severe diabetic ketoacidosis - A comparative study of two methods. Diabetologia. 1979;17(1):17-21.

42. Pfeifer MA, Samols E, Wolter CF, Winkler CF. Low-dose versus high-dose insulin therapy for diabetic ketoacidosis. South Med J. 1979;72(2):149-154. doi:10.1097/00007611-197902000-00012

43. Sacks HS, Shahshahani M, Kitabchi AE, Fisher JN, Young RT. Similar responsiveness of diabetic ketoacidosis to low-dose insulin by intramuscular injection and albumin-free infusion. Ann Intern Med. 1979;90(1):36-42. doi:10.7326/0003-4819-90-1-36

44. Owen OE, Licht JH, Sapir DG. Renal function and effects of partial rehydration during diabetic ketoacidosis. Diabetes. 1981;30(6):510 518. doi:10.2337/diab.30.6.510

45. Basu A, Close CF, Jenkins D, Krentz AJ, Nattrass M, Wright AD. Persisting mortality in diabetic ketoacidosis. Diabetic Med. 1993;10 (3):282-284.

46. Rajasoorya C, Wong SF, Chew LS. Diabetic ketoacidosis-a study of 33 episodes. Singapore Med J. 1993;34(5):381-384.

47. Chu CH, Lee JK, Lam HC, Lu CC The occurrence of diabetic ketoacidosis in type 2 diabetic adults; 1997. Avaliable from: http://wwwtsi morgtw/journal/jour10-6/P10_230PDF. Accessed December 15, 2011.

48. Singh RK, Perros P, Frier BM. Hospital management of diabetic ketoacidosis: are clinical guidelines implemented effectively? Diabetic Med. 1997;14(6):482-486. doi:10.1002/(SICI)1096-9136 (199706)14:6<482::AID-DIA371>3.0.CO;2-A

49. Wagner A, Risse A, Brill H, et al. Therapy of severe diabetic ketoacidosis: zero-mortality under very-low-dose insulin application. Diabetes Care. 1999;22(5):674-677. doi:10.2337/diacare.22.5.674

50. Umpierrez G, Freire AX. Abdominal pain in patients with hyperglycemic crises. J Crit Care. 2002;17(1):63-67.

51. Jabbar A, Farooqui K, Habib A, Islam N, Haque N, Akhter J. Clinical characteristics and outcomes of diabetic ketoacidosis in Pakistani adults with Type 2 diabetes mellitus. Diabetic Med. 2004;21 (8):920-923. doi:10.1111/j.1464-5491.2004.01249.x

52. Newton CA, Raskin P. Diabetic ketoacidosis in type 1 and type 2 diabetes mellitus: clinical and biochemical differences. Arch Intern Med. 2004;164 (17):1925-1931. doi:10.1001/archinte.164.17.1925

53. Lin SF, Lin JD, Huang YY. Diabetic ketoacidosis: comparisons of patient characteristics, clinical presentations and outcomes today and 20 years ago. Chang Gung Med J. 2005;28(1):24-30.

54. Solá E, Garzón S, García-Torres S, Cubells P, Morillas C, Hernández-Mijares A. Management of diabetic ketoacidosis in a teaching hospital. Acta Diabetol. 2006;43(4):127-130. doi:10.100 7/s00592-006-0227-1

55. Ersöz HÖ, Ukinc K, Köse M, et al. Subcutaneous lispro and intravenous regular insulin treatments are equally effective and safe for the treatment of mild and moderate diabetic ketoacidosis in adult patients. Int J Clin Pract. 2006;60(4):429-433. doi:10.1111/j.13685031.2006.00786.x
56. Abela AG, Magri CJ, Debono M, Calleja N, Vassallo J, Azzopardi J. An audit of the management of diabetic ketoacidosis at St. Luke's Hospital. Malta Med J. 2008;20(2):16-21.

57. Kitabchi AE, Murphy MB, Spencer J, Matteri R, Karas J. Is a priming dose of insulin necessary in a low-dose insulin protocol for the treatment of diabetic ketoacidosis? Diabetes Care. 2008;31 (11):2081-2085. doi:10.2337/dc08-0509

58. Huri HZ, Foong GTK, Pendek R, Widodo RT. Different characteristics of diabetic ketoacidosis between type 1 and type 2 diabetes patients in Malaysia. Asian Biomed. 2009;3(2):201-205.

59. Lopes AD, Maciel AT, Park M. Evolutive physicochemical characterization of diabetic ketoacidosis in adult patients admitted to the intensive care unit. J Crit Care. 2011;26(3):303-310. doi:10.1016/j. jcrc.2010.08.013

60. Robles FC, Neto DL, Dias FG, et al. Diabetic ketoacidosis: difference between potassium determined by blood gas analysis versus plasma measurement. Arq Bras Endocrinol Metabol. 2011;55(4):256-259.

61. Al-Rubeaan KA, Aftab SA, Alotaibi MS, Alghamdi AA, Rafiullah MR. Clinico-laboratory characteristics of diabetic keto acidosis in adults in a tertiary hospital in Saudi Arabia. Eur Rev Med Pharmacol Sci. 2011;15(10):1202-1206.

62. Chua HR, Venkatesh B, Stachowski E, et al. Plasma-Lyte 148 vs $0.9 \%$ saline for fluid resuscitation in diabetic ketoacidosis. J Crit Care. 2012;27(2):138-145. doi:10.1016/j.jcrc.2012.01.007

63. Weinert LS, Scheffel RS, Severo MD, et al. Precipitating factors of diabetic ketoacidosis at a public hospital in a middle-income country. Diabetes Res Clin Pract. 2012;96(1):29-34. doi:10.1016/j. diabres.2011.11.006

64. Duhon B, Attridge RL, Franco-Martinez AC, Maxwell PR, Hughes DW. Intravenous sodium bicarbonate therapy in severely acidotic diabetic ketoacidosis. Ann Pharmacother. 2013;47(7-8):970-975. doi:10.1345/aph.1S014

65. Azevedo LCP, Choi H, Simmonds K, Davidow J, Bagshaw SM. Incidence and long-term outcomes of critically ill adult patients with moderate-to-severe diabetic ketoacidosis: retrospective matched cohort study. J Crit Care. 2014;29(6):971-977. doi:10.1016/j. jerc.2014.07.034

66. Seth P, Kaur H, Kaur M. Clinical profile of diabetic ketoacidosis: a prospective study in a Tertiary Care Hospital. J Clin Diagn Res. 2015;9(6):OC01-OC04. doi:10.7860/JCDR/2015/8586.5995

67. Houshyar J, Bahrami A, Aliasgarzadeh A. Effectiveness of insulin glargine on recovery of patients with diabetic ketoacidosis: a randomized controlled trial. J Clin Diagn Res. 2015;9(5):Oc01-Oc05. doi:10.7860/JCDR/2015/12005.5883

68. Usman A, Syed Sulaiman SA, Khan AH, Adnan AS. Profiles of diabetic ketoacidosis in multiethnic diabetic population of Malaysia. Trop J Pharm Res. 2015;14(1):179-185. doi:10.4314/tjpr.v14i1.25

69. Guisado-Vasco P, Cano-Megías M, Carrasco-de la Fuente M, CorresGonzález J, Matei AM, González-Albarrán O. Clinical features, mortality, hospital admission, and length of stay of a cohort of adult patients with diabetic ketoacidosis attending the emergency room of a tertiary hospital in Spain. Endocrinologia Y Nutricion. 2015;62(6):277-284. doi:10.1016/j.endonu.2015.02.003

70. Navarro-Díaz FJ, Amillo M, Rosales M, Panadero A, Ena J. Opportunities to improve hospital emergency care of patients with diabetic ketoacidosis. Emerg. 2015;27(1):39-42.

71. Dhatariya KK, Nunney I, Higgins K, Sampson MJ, Iceton G. National survey of the management of Diabetic Ketoacidosis (DKA) in the UK in 2014. Diabetic Med. 2016;33(2):252-260. doi:10.1111/dme.12875

72. Kakusa M, Kamanga B, Ngalamika O, Nyirenda S. Comatose and noncomatose adult diabetic ketoacidosis patients at the University Teaching Hospital, Zambia: clinical profiles, risk factors, and mortality outcomes. Indian J Endocrinol Metab. 2016;20(2):199-205. doi: $10.4103 / 2230-8210.176358$ 
73. Kamata Y, Takano K, Kishihara E, Watanabe M, Ichikawa R, Shichiri M. Distinct clinical characteristics and therapeutic modalities for diabetic ketoacidosis in type 1 and type 2 diabetes mellitus. $J$ Diabetes Complications. 2017;31(2):468-472. doi:10.1016/j. jdiacomp.2016.06.023

74. Balili CAV, Gomez MHS. Efficacy and safety of subcutaneous insulin analogue versus intravenous insulin infusion among patients with mild to moderate diabetic ketoacidosis at the university of Santo Tomas hospital. Phillippine J Internal Med. 2017;55:1.
75. Chua HR, Schneider A, Bellomo R. Bicarbonate in diabetic ketoacidosis a systematic review. Ann Intensive Care. 2011;1(1):23. doi:10.1186/21105820-1-23

76. Cieluch A, Uruska A, Falkowski B, et al. Nonadherence to potassium replacement protocol leads to prolonged management of diabetic ketoacidosis. Pol Arch Internal Med. 2018;128(78):416-420. doi:10.20452/pamw.4293

\section{Publish your work in this journal}

Diabetes, Metabolic Syndrome and Obesity: Targets and Therapy is an international, peer-reviewed open-access journal committed to the rapid publication of the latest laboratory and clinical findings in the fields of diabetes, metabolic syndrome and obesity research. Original research, review, case reports, hypothesis formation, expert opinion and commentaries are all considered for publication. The manuscript management system is completely online and includes a very quick and fair peer-review system, which is all easy to use. Visit http://www.dovepress.com/testimonials.php to read real quotes from published authors. 